\title{
HISTOLOGY OF TUBERCULOSIS OF INTESTINES AND LIVER.
}

Br E. J. McWEENEY, M.D.;

Pathologist to the Mater Misericordiø Hospital ;

Professor of Pathology and Bacteriology, Catholic Dniversity

Medical School.

[Read in the Section of Pathology, February 23, 1900.]

Professor E. J. McWeeney showed some naked-eye and microscopical preparations of this condition taken from a patient in Dr. Dempsey's ward at the Mater Misericordiæ Hospital, who had died of diarrboa due to tuberculous enteritis. The floor of the very extensive ulcers was composed of granulation tissue consisting of cells with simple and polymorphous nuclei, but seemingly devoid of giant cells. Enormous multitudes of tubercle bacilli were lying between these cells, and were often actually in contact with, but not within, them; yet the tissue showed practically no sign of caseation-a remarkable fact in view of the extensive areas of caseation met with so commonly in tuberculous lymphatic glands, where even the most careful examination fails to reveal a single bacillus. The toxin that produces caseation might conceivably be contained within the bacillary bodies, and only becomes free by their breakdown. This, at any rate, would be an explanation of the frequent want of association between demonstrable bacilli and extensive caseation. The liver sections constituted a classic object for the study of the histogenesis of tubercle, shorving as they did tubercles in the very earliest stage of development, when they consisted of only a few epithelioid and lymphoid nuclei. As the result of a very careful examination of a number of sections, exhibitor had arrived at the 
conclusion that many of the tubercles began by proliferation of the nuclei of intra-lobular capillary endothelium. The products of such proliferation were the "epithelioid" cells, with which a certain number of uninucleated "lymphoid" elements were $a b$ initio associated. A certain amount of fibrin was present from the very commencement, and formed a reticulum between the cells of the young tubercle. The first effect on the surrounding liver cells was compression and flattening. As the result of careful study he had arrived at the conclusion that the hepatic cells immediately adjoining the tubercle underwent metaplasia, and became converted into "epithelioid" elements. Giant cells began to make their appearance when the tubercle attained a diameter of $0.2 \mathrm{~mm}$; ; between 0.1 and $0.2 \mathrm{~mm}$. giant cells were absent. They were at first no bigger than an epithelioid cell, but were characterised by a more distinct margin and a plurality of nuclei, which were the closer packed the younger the giant cell. He had convinced himself that, in this case at least, the multiplication of nuclei took place either by direct division or by a sort of sprouting. He had failed to observe mitoses in young giant cells.

Dr. A. C. O'Sullifyan said if he understood the first experiment on the typhoid bacillus rightly, it was really decrease of acidity that was being estimated. It would be interesting to know if a further condition of alkalinity would make the growth go off, or possibly make a stronger growth. The observation that phenol acted first on the anaerobic growths might be explained by the fact that typhoid bacilli are what are called facultative anaerobes-that is, their natural mode of growth is aerobic, and their anaerobic growth is, therefore, strained, and the inhibitory drug acts first on the restrained kind of growth. He could corroborate Dr. McWeeney's results as regards Parietti's solution, as he had frequently observed that $B$. coli will grow in 
Parietti's broth in stronger solutions than typhoid-that is to say, if they had two sets of tubes side by side, the typhoid tube would remain clear, while the $B$. coli tube of the same strength would produce a growth. The method of Parietti was very useful for the analysis of water, but was entirely useless for analysing milk for the typhoid bacillus, because there were so many organisms in milk standing a mixture of hydrochloric acid and carbolic acid better than the typhoid bacillus. Speaking of the tubercular lesions examined, he said the case in which he found more tubercle bacilli than in other cases was a case of rery acute pulmonary tuberculosis, a rather rare form, in which the lung was invaded by an exudation, mainly serous, and on inspection the lung did not appear as if tuberculous at all. The case occurred in a child, and death was very rapid. There were no tubercles, no giant cells, no caseation, but the amount of tubercle bacilli found in the alveoli was enormous, so that in that case a rapid course seemed to have been produced by a large number of bacilli.

DR. E. J. McW EENET, in reply, said he agreed with the statement that Parietti's solution was of no use for the analysis of milk. On plating out with gelatine they got a number of things which they could not say definitely to be true $B$. coli or pseudo-coli. He acknowledged the justice of the correction with regard to the reaction - he should hare said decrease of acidity. 